\title{
The maize Brown midrib1 locus affects cell wall composition and plant development in a dose- dependent manner
}

\author{
W Vermerris ${ }^{1,2}$, KJ Thompson ${ }^{1}$ and LM McIntyre ${ }^{1,3}$ \\ ${ }^{1}$ Department of Agronomy, Purdue University, West Lafayette, IN 47907, USA; ${ }^{2}$ Department of Agricultural and Biological \\ Engineering, Purdue University, West Lafayette, IN 47907, USA; ${ }^{3}$ Computational Genomics Group, Purdue University, West \\ Lafayette, IN 47907, USA
}

The four brown midrib $(\mathrm{bm})$ mutants of maize have a reduced content and altered subunit composition of the cell wall polymer lignin. The $\mathrm{bm}$ mutations have traditionally been considered completely recessive, because the brown midrib phenotype is only apparent in plants homozygous for the mutation. In addition to an effect on cell wall composition, some $\mathrm{bm}$ mutations have been shown to affect flowering time. We had preliminary evidence for a dosage effect of the $\mathrm{Bm} 1$ locus on flowering time, which prompted this detailed study on the $B m 1$ locus. In this study, near-isogenic lines (in an A619 background) with zero, one or two bm1 mutant alleles were compared. The $b m 1$ heterozygotes flowered significantly earlier than both the wild-type plants and $\mathrm{bm} 1$ mutants. This difference can at least be partly attributed to an accelerated growth rate in the later stages of plant development. Furthermore, Fourier transform infrared spectroscopy revealed that the cell wall composition of the $b m 1$ heterozygous plants is distinct from both the $b m 1$ and wildtype homozygotes. The combination of the data on flowering time and the data on cell wall composition provide evidence for a dosage effect at the $B m 1$ locus.

Heredity (2002) 88, 450-457. DOI: 10.1038/sj/hdy/6800078

Keywords: brown midrib; cell wall; flowering; maize; pollen; Zea mays

\section{Introduction}

The four brown midrib (bm) mutants of maize (Zea mays L.) have brown vascular tissue in the leaf and stem, which is related to a reduction in content and change in subunit composition of the cell wall polymer lignin (Kuc et al, 1968). Lignin is important for the survival of the plant because of its role in water transport, rigidity and defense against pests and pathogens. On the other hand, the presence of lignin has been associated with reduced forage quality (Cherney et al, 1991). The lignin monomers, $p$ coumaryl alcohol, coniferyl alcohol and sinapyl alcohol, are synthesized via the concerted action of the shikimic acid and phenylpropanoid pathways. After synthesis and transport to the cell wall these monomers undergo polymerization via an oxidative coupling mechanism. Recent progress in understanding lignin biosynthesis has been reviewed by Baucher et al (1998) and Whetten et al (1998).

The Brown midrib genes play a role in the biosynthesis of lignin, and analyzing the lignin of the $b m$ mutants is helpful in understanding the role of the functional genes and the proteins they encode. The $b m$ mutants offer the possibility to increase understanding of lignification and to help identify how changes in lignin affect other parts of plant metabolism. The bm1 mutant incorporates coniferaldehyde residues in the cell wall and stains more

Correspondence: W Vermerris, Department of Agronomy, Purdue University, West Lafayette, IN 47907, USA. E-mail: vermerris@purdue.edu Received 10 April 2001; accepted 9 January 2002 brightly in the Wiesner reaction (Provan et al, 1997; Halpin et al, 1998). The Wiesner reaction is based on the formation of a red chromophore resulting from the reaction between phloroglucinol and coniferaldehyde end groups under acidic conditions (Geiger and Fuggerer, 1979; Provan et al, 1997). The changes in lignin composition in the $b m 1$ mutant are correlated with a reduced activity of the enzyme cinnamyl alcohol dehydrogenase (CAD) (Halpin et al, 1998). The bm2 mutant has a reduction in guaiacyl residues (Chabbert et al, 1994) and a disturbance in the tissue-specific patterns of lignification (Vermerris and Boon, 2001). The bm3 mutant accumulates 5-hydroxyguaiacyl residues in its lignin (Lapierre, 1993) as a result of a mutation in the gene encoding caffeic acid O-methyltransferase (Vignols et al, 1995). The bm4 mutant is the least characterized brown midrib mutant and little is currently known about its chemical composition. All four brown midrib mutations are considered to be completely recessive (Neuffer et al, 1997), since the brown midrib phenotype is only visible in plants that are homozygous for the mutant allele.

The $b m$ mutations have been shown to affect flowering time (Vermerris and McIntyre, 1999). The bm2 mutation delayed flowering time, while the $b m 1$ mutation tended to expedite it. There is additional evidence for a relationship between cell wall composition and flowering. Maize plants with the bm3 mutation were reported to flower later than their wild-type counterparts (Gentinetta et al, 1990) and a quantitative trait loci (QTL) affecting flowering time was identified near the bm4 locus (Koester et al, 1993). A survey of the Maize Genetics Database 
(www.agron.missouri.edu) reveals that QTL for flowering time map near all four maize brown midrib loci.

In trying to understand the relationship between lignin biosynthesis and flowering several possibilities need to be considered. Lignin content per se is an unlikely factor, since the $b m 1-b m 2$ double mutant, which has the lowest lignin content among the genotypes that were analyzed, did not show significant changes in flowering time (Vermerris and McIntyre, 1999). It is, however, possible that flowering time is influenced by lignin composition. As lignin plays a role in water transport, it is possible that certain alterations in lignin composition can mimic drought stress. The water potential is known to affect flowering dynamics. In addition to a reduction in plant height (Sari-Gorla et al, 1999), drought conditions tend to result in increased silking delay (Moss and Downey, 1971; Herrero and Johnson, 1981). The silking delay, or anthesis-silking interval (ASI), refers to the time that elapses between pollen shedding and the appearance of silks on the ear of the same plant. This is the result of a reduction in the time to anthesis, and an increase in the time to silking (Ribaut et al, 1996; Veldboom and Lee, 1996). Alterations in lignin composition that would simulate drought stress are therefore anticipated to decrease flowering time and increase silking delay.

Another possibility is that variation in flowering time is the result of changes in growth and development prior to flowering. For example, an accelerated growth rate would expedite the overall maturation of the plant, including flowering.

In this study the role of the Bm1 locus on growth and development and on cell wall composition was examined by comparing near-isogenic lines with zero, one or two bm1 mutant alleles. These experiments were designed to begin to understand the nature of the relationship between cell wall biogenesis and flowering.

\section{Materials and methods}

\section{Plant material}

The bm1 seed was derived from a seed stock that also contained the linked mutations anthocyaninless2 (a2) and purple aleuronel ( $p r 1)$. This seed stock was kindly provided by Dr RL Phillips (University of Minnesota, St Paul, MN, USA) and represented $\mathrm{BC}_{6} \mathrm{~F}_{1}$ seed in an A619 background. A bm1 near-isogenic line (NIL) in an A619 background was produced from this stock by additional backcrossing. The recessive mutations $a 2$ and $p r 1$ affect the color of the seed. The $a 2-b m 1-p r 1 \quad \mathrm{BC}_{6} \mathrm{~F}_{1}$ seeds were yellow. Ten plants grown from these seeds were backcrossed to A619 (female), which resulted in all purple $\mathrm{BC}_{7} \mathrm{~F}_{1}$ kernels. Thirty $\mathrm{BC}_{7} \mathrm{~F}_{1}$ plants (heterozygous for all three loci) were grown from this seed. All 30 plants were self pollinated, resulting in seeds that were segregating for kernel color (yellow, purple, and purple seeds with brown pericarps). The yellow seeds were either a2pr1 or wild-type. In order to identify $b m 1$ mutants flanked by the wild-type alleles of $A 1$ and $\operatorname{Pr} 1,200$ yellow $\mathrm{BC}_{7} \mathrm{~F}_{2}$ seeds (from three $\mathrm{BC}_{7} \mathrm{~F}_{1}$ plants) were planted. The $b m 1$ mutants among them were identified, self pollinated and crossed to inbred line A619. The majority of the $b m 1$ plants produced colored seeds from one or both of these crosses, but five bm1 plants produced all yellow seeds from both the self pollination and the cross to A619, and were $b m 1$ NILs. The seed produced from the self pollination was used for the analysis of $b m 1$ homozygotes, whereas the seeds from the cross to A619 were used as $b m 1$ heterozygotes. The same seed sources were used in all experiments.

\section{Field design}

Twenty seeds of each of the three genotypes, wild type, $b m 1 /+$, and $b m 1$, were planted in a Latin square the summer of 1999 in Clayton, NC (Latin square I). Fifty-two plants reached anthesis. Two Latin squares containing the same three genotypes were planted in the summer of 2000 in Throckmorton, IN (Latin square IIA, B) where 48 and 50 plants, respectively, reached anthesis. The plants were irrigated and fertilized according to standard nursery procedures.

\section{Data collection}

Flowering time (anthesis) and silking time were recorded by daily survey of individual plants and measured in days after planting. Flowering was defined as the emergence of pollen-shedding anthers from the tassel, and silking was defined as the emergence of the first silks from the ear. The ASI was defined as the time difference (in days) between flowering time and silking time. The height at anthesis was recorded for each individual plant. The development stage for each individual plant in Latin Square IIA and B was recorded every other day using the method described in Special Report no. 48 (Iowa State University Cooperative Extension Service, 1997). Staging data were recorded beginning at stage V2. Two data collection days were missed, one due to pesticide treatment and another due to inclement weather.

\section{Statistical analysis}

The flowering time, silking time, and time to each developmental stage were modeled using survival analysis as described by Vermerris and McIntyre (1999). In a survival analysis the time to an event is modeled without assumption of normality for the outcome. In the case of flowering time and time to developmental stages the distribution was often skewed. In order to carry out an analysis of variance (ANOVA), the data were log-transformed and then the log of the time-to-event was modeled. The ASI was normally distributed and was modeled using an ANOVA. All statistical analyses were performed using the SAS software package (SAS Institute, Cary, NC, USA).

\section{Fourier-transform infrared spectroscopy}

Fourier transform infrared (FT-IR) spectroscopy was performed in order to compare the chemical composition of the cell walls of the three genotypes. FT-IR spectroscopy is a method that relies on the absorption of energy from infrared light as a result of changes in the vibration energy of chemical bonds. These vibrations can either involve the distance between the atoms (stretch) or the angle between the atoms (bend), but can only be detected with FT-IR spectroscopy if they also involve a net change in the dipole moment. The difference in signal between the transmitted and detected light at different frequencies can be represented in a spectrum, and thus represents information on the chemical composition of the sample. Various functional groups have characteristic absorbances and their presence can be used for diagnostic 
purposes. FT-IR spectra of ligno-cellulosic materials are typically complex, because of overlap in signal between various functional groups (Faix, 1992; Séné et al, 1994; Stewart et al, 1997).

Among several different analytical methods available to characterize cell wall composition FT-IR spectroscopy was selected, because it is uniquely suitable to assay quantitative variation between cell wall samples without chemical extraction of particular cell wall fractions. Furthermore, FT-IR spectroscopy can also detect changes in interactions between cell wall compounds even if the actual concentrations of particular compounds are not altered (Séné et al, 1994). Furthermore, the analysis requires only small amounts of tissue.

When the tassel had just emerged (VT stage), vascular tissue from the midrib of leaf 6 was dissected with a scalpel and cut in sections of $3-5 \mathrm{~mm}$. The sections were soaked in water $(2 \times 30 \mathrm{~min})$ to remove water-soluble compounds, and in acetone $(2 \times 30 \mathrm{~min})$ to remove unbound phenolic compounds. Samples of 2.0, 3.5 and $5.0 \mathrm{mg}$ of dissected wild-type tissue were ground, mixed with $250 \mathrm{mg}$ dry $\mathrm{KBr}$ powder and pressed into a thin pellet. The FT-IR spectrum was recorded using a Spectrum GX FT-IR spectrometer (Perkin Elmer; Norwalk, CT, USA). A pellet of $250 \mathrm{mg} \mathrm{KBr}$ without maize tissue was used as a blank. Nitrogen gas was used in the sample chamber and detector to warrant a consistent atmosphere. A total of 64 scans were recorded between 4000 and $370 \mathrm{~cm}^{-1}$ at a resolution of $2 \mathrm{~cm}^{-1}$. In the spectrum of the $2.0 \mathrm{mg}$ sample the peak resolution was not as good as in the spectrum of the $3.5 \mathrm{mg}$ sample. The $5.0 \mathrm{mg}$ sample produced a spectrum that looked saturated. The comparisons between genotypes were therefore made with samples of $3.5 \mathrm{mg}$.

The midribs of two plants of each genotype were analyzed in duplicate. The data were acquired using Spectrum v. 3.02 software (Perkin Elmer) and analyzed with the Grams v.6.0 software package (Galactic Industries, Salem, NH, USA). Comparisons between spectra (in the range between 1800 and $750 \mathrm{~cm}^{-1}$ ) of the three genotypes were based on spectra that were baseline corrected and area-normalized. An ANOVA was used to evaluate overall differences in the absorbance at several diagnostic frequencies.

\section{Wiesner reactions}

Wiesner reactions were performed by incubating dissected midribs in $0.2 \mathrm{ml} 1 \%(\mathrm{w} / \mathrm{v})$ phloroglucinol in ethanol:concentrated $\mathrm{HCl}$ (3:1). After $10 \mathrm{~min}$ incubation the supernatant was removed and the samples were rinsed in water.

\section{Results}

Maize plants heterozygous at the $B m 1$ locus flower earlier than their homozygous counterparts

Table 1 shows the mean number of days until each developmental stage, flowering time, and silking time for Latin Square IIA and IIB. Mean flowering time in Latin square I for $\mathrm{A} 619, b m 1$ and $b m 1 /+$ was $74.8,73.7$, and 71.5 days after planting, respectively, with a standard deviation of $2.77,1.16$, and 1.36 days. Survival analyses were performed to compare flowering time and silking time of the three genotypes. Hazard ratios appeared to be pro- portional, allowing the use of the semi-parametric Coxproportional hazards model. The parametric models using a log-logistic, log-normal, and generalized gamma distribution were also considered. Table 2 summarizes the results from the flowering and silking time analysis. Based on the log-likelihood values the three parametric models perform equally well. The $P$-values for the test of equality of genotypes ( $b m 1$ compared with wild type, and $b m 1 /+$ compared with wild type) using the different survival models, and the ANOVA results for log-transformed flowering time are also listed in Table 2. From these results it is clear that the heterozygous plants are flowering earlier than the wild-type plants. It is noteworthy that this is irrespective of the model that was chosen. Additional contrasts were conducted to test whether the $b m 1$ heterozygotes also flowered earlier than the bm1 homozygotes. In most models this difference was not statistically significant (data not shown). A graphic representation of the predicted survival curves shows a similar pattern for all models. Figure 1 shows the predicted survival curves of the wild-type, $b m 1$ and $b m 1 /+$ plants from the three replicates using the log-logistic survival model. In all three replicates the predicted survival curves show that $b m 1$ heterozygote flowers earlier than the $b m 1$ mutant and the $b m 1$ mutant flowers earlier than the wild type. The difference between the $b m 1$ mutant and the wild type is statistically significant in Latin Square IIA. According to some models the silking time of the $b m 1 /+$ plants is significantly earlier than the wildtype plants in Latin square IIB (P-values 0.01-0.051), but not in Latin square IIA ( $P$-values $0.41-0.78)$. The silking time of the $b m 1$ mutants is not significantly different from the wild-type plants in either of the two replicates. Despite the lack of statistically significant differences, silking time follows the same basic pattern as the flowering time.

\section{The $b m 1$ heterozygote has a distinct cell wall composition}

Figure 2 shows dissected midribs of wild-type, $b m 1 /+$ and $b m 1$ plants. The $b m 1$ samples have a distinct brown color relative to the yellow color of the wild-type samples. The $b m 1 /+$ samples look similar to the wildtype samples. After incubation of the samples with the Wiesner reagent the $b m 1$ samples are stained more intensely than the wild-type and $b m 1 /+$ samples. This indicates that the cell wall of the $b m 1$ mutant contains higher levels of coniferaldehyde residues. The results from the Wiesner reaction are consistent with the notion that the bm1 mutation is recessive (Neuffer et al, 1997).

The fact that the $b m 1$ heterozygotes flower earlier than both homozygotes suggests a dosage effect. The Wiesner reaction is primarily a qualitative assay, and despite lack of variation in color prior to and after staining with the Wiesner reagent between the wild type and $b m 1$ heterozygotes, the $b m 1$ heterozygotes may have a different cell wall composition. A chemical analysis of the cell walls was conducted to ascertain whether the cell walls were indeed different among the three genotypes. Figure 3 displays the baseline-corrected, area-normalized and averaged FT-IR spectra of the cell wall samples from the three genotypes in the range between 1800 and $750 \mathrm{~cm}^{-1}$. It is illustrative to compare the overall spectra of the wild type and $b m 1$ mutant first. These two genotypes have been shown to differ in cell wall composition (Provan et 
Table 1 Means and standard deviations (SD) for the time to each developmental stage, flowering time, and silking time (in days after planting) in Latin squares IIA and IIB. The asterisks indicate missing data

\begin{tabular}{|c|c|c|c|c|c|c|c|c|c|c|c|c|}
\hline \multirow[t]{3}{*}{ Stage } & \multicolumn{6}{|c|}{ Latin square IIA } & \multicolumn{6}{|c|}{ Latin square IIB } \\
\hline & \multicolumn{2}{|c|}{ wild type } & \multicolumn{2}{|c|}{$b m 1 /+$} & \multicolumn{2}{|c|}{$b m 1 / b m 1$} & \multicolumn{2}{|c|}{ wild type } & \multicolumn{2}{|c|}{$b m 1 /+$} & \multicolumn{2}{|c|}{$b m 1 / b m 1$} \\
\hline & time (dap) & $S D$ & time (dap) & $S D$ & time (dap) & $S D$ & time (dap) & $S D$ & time (dap) & $S D$ & time (dap) & $S D$ \\
\hline $\mathrm{V} 2$ & 23.4 & 3.4 & 22.7 & 1.8 & 23.5 & 2.8 & 18.4 & 0.8 & 18.2 & 0.6 & 18.7 & 2.2 \\
\hline V3 & 29.9 & 1.5 & 29.0 & 0.0 & 29.3 & 1.0 & 20.5 & 0.9 & 20.1 & 0.5 & 21.1 & 2.4 \\
\hline $\mathrm{V} 4$ & 32.1 & 2.0 & 31.0 & 0.0 & 31.7 & 1.5 & 23.0 & 1.6 & 22.4 & 1.4 & 22.9 & 2.3 \\
\hline V5 & 35.0 & 3.3 & 33.9 & 1.0 & 33.8 & 1.8 & 27.3 & 1.3 & 27.1 & 1.5 & 28.3 & 1.3 \\
\hline V6 & 37.3 & 2.7 & 37.5 & 1.2 & 37.7 & 2.9 & 30.0 & 0.0 & 30.0 & 2.1 & 30.1 & 0.5 \\
\hline V7 & 44.8 & 2.7 & 45.2 & 2.3 & 45.3 & 2.1 & 40.2 & 3.5 & 39.7 & 2.2 & 40.8 & 1.7 \\
\hline V8 & 52.0 & 5.1 & 48.2 & 1.6 & 49.5 & 3.3 & 45.9 & 2.0 & 45.0 & 1.0 & 46.4 & 3.1 \\
\hline V9 & 57.3 & 5.7 & 54.9 & 5.9 & 54.8 & 5.6 & * & $*$ & 51.0 & 4.2 & 54.8 & 1.8 \\
\hline VT & 68.1 & 2.5 & 65.6 & 1.3 & 66.5 & 1.8 & 59.1 & 2.7 & 57.4 & 1.2 & 58.6 & 2.2 \\
\hline Flower & 70.2 & 3.0 & 67.2 & 1.5 & 68.0 & 2.3 & 60.3 & 1.7 & 58.9 & 1.2 & 59.8 & 1.7 \\
\hline Silk (R1) & 72.2 & 3.2 & 71.2 & 3.1 & 71.4 & 3.1 & 62.2 & 3.0 & 60.1 & 1.7 & 61.1 & 2.6 \\
\hline
\end{tabular}

Table 2 Comparison of different models to estimate differences in flowering time and silking time for the $b m 1 / b m 1$ and $b m 1 /+$ plants compared with the wild-type plants

\begin{tabular}{|c|c|c|c|c|c|c|c|}
\hline \multirow[t]{2}{*}{ Replicate } & \multirow[t]{2}{*}{ Model } & \multicolumn{3}{|c|}{ Flowering } & \multicolumn{3}{|c|}{ Silking } \\
\hline & & Log-likelihood & genotype & P-value & Log-likelihood & genotype & P-value \\
\hline I & $\begin{array}{l}\text { Log-logistic } \\
\text { Log-normal } \\
\text { Gamma } \\
\text { Cox } \\
\text { GLM* }\end{array}$ & $\begin{array}{l}6 \begin{array}{r}63.15 \\
61.87\end{array} \\
\text { failed to converge }\end{array}$ & $\begin{array}{l}b m 1 / b m 1 \\
b m 1 /+ \\
b m 1 / b m 1 \\
b m 1 / /+ \\
b m 1 / b m 1 \\
b m 1 / /+ \\
b m 1 / b m 1 \\
b m 11 /+ \\
b m 1 / b m 1 \\
b m 1 /+\end{array}$ & $\begin{array}{r}0.2975 \\
<0.0001 \\
0.1302 \\
<0.0001 \\
\\
\\
0.2606 \\
<0.0001 \\
0.0923^{\mathrm{a}} \\
<0.0001^{\mathrm{a}}\end{array}$ & & & \\
\hline IIA & $\begin{array}{l}\text { Log-logistic } \\
\text { Log-normal } \\
\text { Gamma } \\
\text { Cox } \\
\text { GLM* }\end{array}$ & $\begin{array}{r}34.04 \\
34.15 \\
35.68 \\
316.77\end{array}$ & $\begin{array}{l}b m 1 / b m 1 \\
b m 1 /+ \\
b m 1 / b m 1 \\
b m 1 /+ \\
b m 1 / b m 1 \\
b m 1 / /+ \\
b m 1 / b m 1 \\
b m 1 /+ \\
b m 1 / b m 1 \\
b m 1 /+\end{array}$ & $\begin{array}{l}0.0473 \\
0.0103 \\
0.0259 \\
0.0051 \\
0.0245 \\
0.0173 \\
0.4556 \\
0.0378 \\
0.0367 \\
0.0096\end{array}$ & $\begin{array}{r}24.59 \\
25.64 \\
25.69 \\
702.71\end{array}$ & $\begin{array}{l}b m 1 / b m 1 \\
b m 1 /+ \\
b m 1 / b m 1 \\
b m 11 /+ \\
b m 1 / b m 1 \\
b m 11 /+ \\
b m 1 / b m 1 \\
b m 1 /+ \\
b m 1 / b m 1 \\
b m 1 /+\end{array}$ & $\begin{array}{l}0.7893 \\
0.7860 \\
0.6553 \\
0.5534 \\
0.6380 \\
0.5342 \\
0.3632 \\
0.4089 \\
0.6682 \\
0.5696\end{array}$ \\
\hline IIB & $\begin{array}{l}\text { Log-logistic } \\
\text { Log-normal } \\
\text { Gamma } \\
\text { Cox } \\
\text { GLM }^{*}\end{array}$ & $\begin{array}{r}28.97 \\
27.29 \\
29.22 \\
283.94\end{array}$ & $\begin{array}{l}b m 1 / b m 1 \\
b m 1 /+ \\
b m 1 / b m 1 \\
b m 1 /+ \\
b m 1 / b m 1 \\
b m 11 /+ \\
b m 1 / b m 1 \\
b m 1 /+ \\
b m 1 / b m 1 \\
b m 11 /+\end{array}$ & $\begin{array}{l}0.1570 \\
0.0010 \\
0.2994 \\
0.0022 \\
0.3254 \\
0.0017 \\
0.1090 \\
0.0271 \\
0.3195 \\
0.0047\end{array}$ & $\begin{array}{c}10.84 \\
10.99 \\
14.04 \\
307.86\end{array}$ & $\begin{array}{l}b m 1 / b m 1 \\
b m 11 /+ \\
b m 1 / b m 1 \\
b m 1 /+ \\
b m 1 / b m 1 \\
b m 11 /+ \\
b m 1 / b m 1 \\
b m 11 /+ \\
b m 1 / b m 1 \\
b m 1 /+\end{array}$ & $\begin{array}{l}0.2174 \\
0.0175 \\
0.2442 \\
0.0063 \\
0.8258 \\
0.0511 \\
0.4801 \\
0.0480 \\
0.2646 \\
0.0109\end{array}$ \\
\hline
\end{tabular}

*The ANOVA was performed on the log-transformed data. The $P$-values in this case are different from the $P$-values for the survival analysis in that they refer to differences between means rather than a comparison of hazard ratios.

${ }^{a}$ Residuals do not appear normally distributed. 

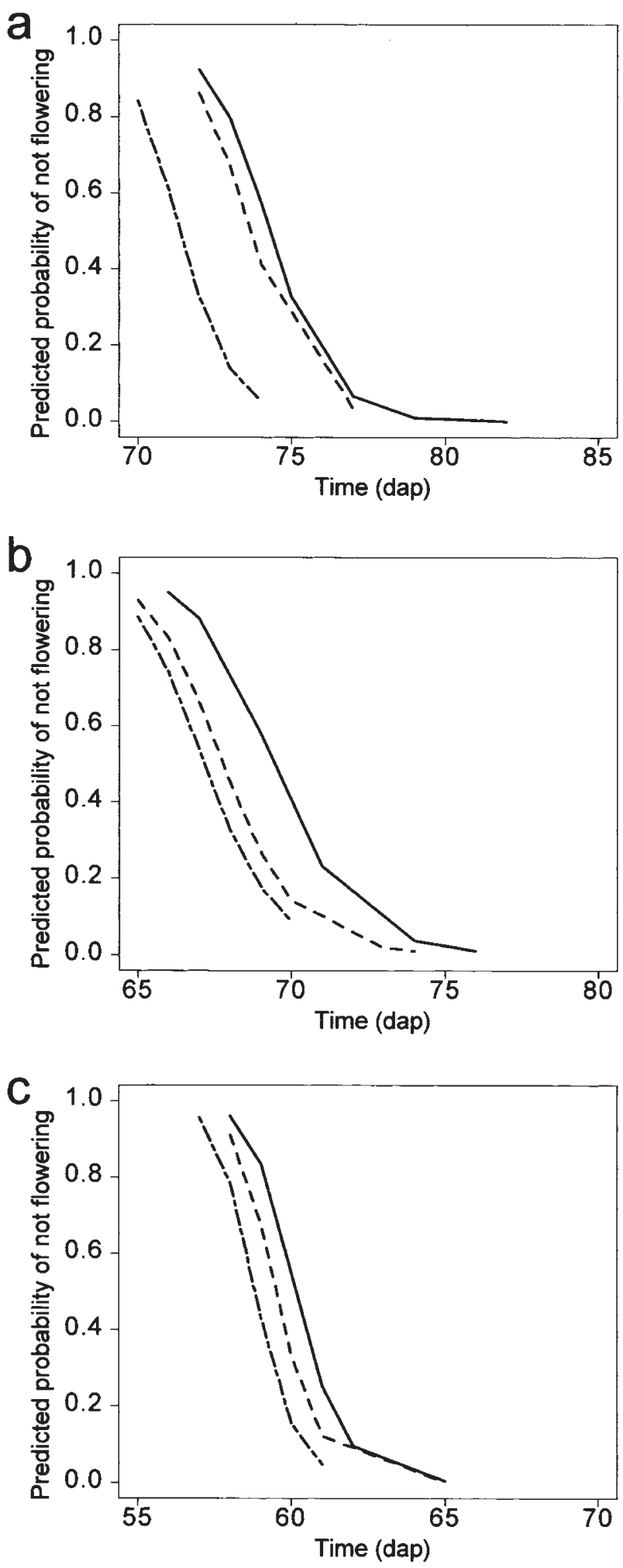

Figure 1 Predicted probability of not flowering using the log-logistic survival model for the wild-type plants (solid line), $b m 1 / b m 1$ plants (dashed line), and $b m 1 /+$ plants (dot-dashed line) in (a) Latin square I, (b) Latin square IIA and (c) Latin square IIB. Time is indicated in days after planting (dap).

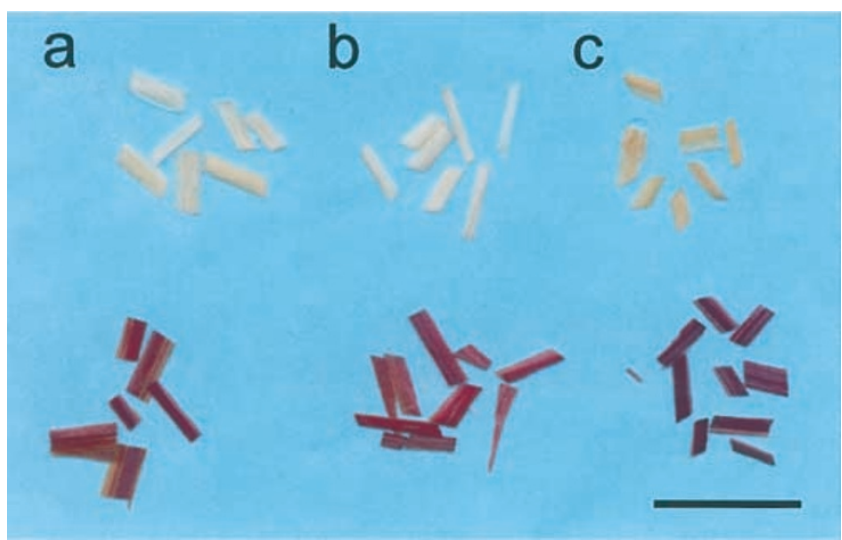

Figure 2 Dissected midribs from leaf 6 of (a) wild-type, (b) $b m 1 /+$ and (c) $b m 1 / b m 1$ plants prior to (top panel) and after (bottom panel) staining with the Wiesner reagent. The midribs of the $b m 1$ mutant are amber-brown, unlike the midribs of the $b m 1$ heterozygote. The midribs of the $b m 1$ mutant display a more intense staining with the Wiesner reagent, whereas the midribs from the wild type and $b m 1$ heterozygote are similar. The bar indicates a length of $1 \mathrm{~cm}$.

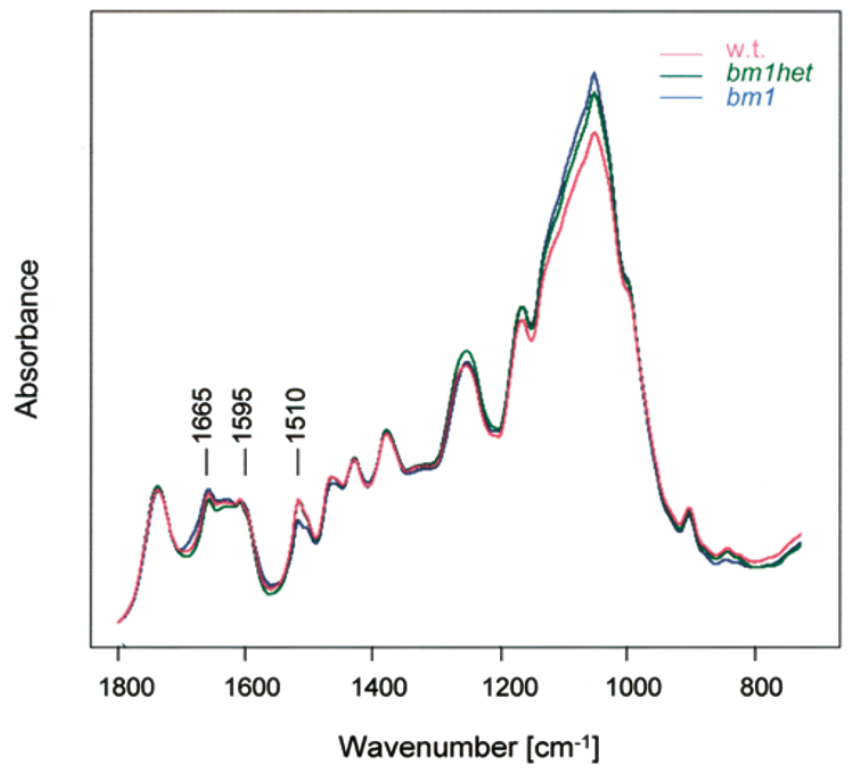

Figure 3 Averages of baseline-corrected, area-normalized FT-IR spectra of dissected midribs of wild type, $b m 1 /+$ and $b m 1 / b m 1$ plants.

al, 1997; Halpin et al, 1998) and therefore provide an indication of the magnitude of changes that can be expected. It is apparent that there is only limited variation between those two spectra, which indicates that the overall cell wall composition and architecture of the bm1 mutant is not drastically different from that of the wild type.

Several differences between the three spectra are apparent. The peak at $1665 \mathrm{~cm}^{-1}$ represents $\mathrm{C}=\mathrm{O}$ stretching in the carbonyl moiety of conjugated aldehydes or ketones (Mackay et al, 1997; Stewart et al, 1997) and based on an ANOVA the difference in absorbance that is observed for the three genotypes is statistically significant $(P=0.0004)$. The peaks at 1595 and $1510 \mathrm{~cm}^{-1}$ are indicative of lignin and hydroxycinnamic acids (Stewart et al, 1997). The value of $\mathrm{A}_{1510}$ of the $b m 1$ mutant is con- 
siderably lower compared with the wild type and $b m 1$ heterozygote and there is a statistically significant difference between genotypes $(P=0.0007)$. A high ratio of $A_{1665}: A_{1510}$ and $A_{1595}: A_{1510}$ is indicative of the incorporation of coniferaldehyde residues in the cell wall (Mackay et al, 1997; Stewart et al, 1997). Both ratios are higher in the bm1 mutant, confirming the results from Wiesner reactions (Figure 2). The ANOVA comparison indicates that the three genotypes are different $(P<0.0001)$. The peak at $1250 \mathrm{~cm}^{-1}$ reflects $\mathrm{C}-\mathrm{O}$ stretching of phenolics (Séné et al, 1994). The difference between the genotypes at this frequency is significant $(P=0.02)$ and the $b m 1$ heterozygote appears to have an absorbance that is distinct from the two homozygotes. The large peak with a maximum at $1048 \mathrm{~cm}^{-1}$ is due to the presence of polysaccharides (Séné et al, 1994; Stewart et al, 1997). Each of the three genotypes appears to have a distinct absorbance, and the overall difference between the three genotypes is statistically significant $(P=0.003)$. The spectrum of the $b m 1$ heterozygote is similar to that of the wild-type at some frequencies (for example $1510 \mathrm{~cm}^{-1}$ ), but more similar to that of the $b m 1$ mutant at other frequencies (for example $1150 \mathrm{~cm}^{-1}$; absorbance different from wild type $(P=0.003))$. At several other frequencies (for example 1250 and $1048 \mathrm{~cm}^{-1}$ ) the spectrum of the $b m 1$ heterozygote appears to show distinct absorbances. Therefore, based on these FT-IR data there is evidence that the $b m 1$ heterozygote displays a chemical phenotype that is distinct from both homozygotes.

\section{There is no evidence for mimicked drought stress}

In order to test whether the changes in cell wall composition mimicked drought stress and thus resulted in an increase in the anthesis-silking interval and/or a decrease in plant height, the silking time and plant height at anthesis were recorded. Table 3 lists the ASI and the mean plant height for the wild type, $b m 1$ and $b m 1 /+$ plants. An ANOVA with ASI as the outcome did not provide evidence for a difference between genotypes. This indicates that the silking delay is not affected by the $b m 1$ mutation, under the conditions tested. There was limited evidence for significant differences in height among the three genotypes. In Latin square IIA the differences in plant height at flowering between the wild-type plants and the $b m 1$ heterozygotes were significantly different. The wild-type plants were the tallest, followed by the $b m 1$ mutants and then the $b m 1$ heterozygotes. The ranking of tallest to shortest genotype, however, was different between the two replicates. In Latin square IIB the $b m 1$ mutants were the tallest followed closely by the wild types and then the $b m 1$ heterozygotes. This difference was not significant, suggesting that the $b m 1$ mutation does not have a significant effect on plant height under the conditions tested.

The differences in ASI and plant height are not consistent across replications. This indicates that the change in cell wall composition does not result in a mimicked drought stress under the conditions tested.

\section{Growth and development in the early stages are not affected by the $b m 1$ mutation}

The differences in flowering time may be the result of an overall change in growth and development of the plant. It is also possible that the flowering time is controlled directly. Under the latter scenario the different genotypes would grow at the same rate until tassel formation and anthesis. Comparing the time it takes a plant to reach a specific developmental stage can distinguish between these two possibilities. The time to each of the developmental stages was recorded every other day for each of the plants in Latin square IIA and IIB and compared using survival analyses at each stage. The results of this analysis indicate that until stage V7 there is no significant difference in growth and development between the three genotypes. During and after stage V8 the $b m 1 /+$ plants seem to grow at a higher rate. The current data, however, are not sufficient to attribute the earlier flowering of the $b m 1 /+$ plants solely to this accelerated growth in or after V8 for two reasons. First, the development of the plants is quite rapid at this stage and changes can occur daily. Second, a crucial day was missed due to pesticide treatment during the transition from V8. In order to determine whether growth after V7 is different, it will be necessary to record the growth and development daily.

\section{Discussion}

Like many mutations the maize brown midrib mutations have been considered recessive, referring to the fact that the mutant phenotype is only apparent in plants homozygous for the mutant allele. While this is true for the brown color of the vascular tissue in the $b m 1$ mutant, we hypothesized the existence of a dosage effect at the $B m 1$ locus to explain the fact that in our previous study the segregating population (with wild-type plants and $b m 1$ heterozygotes) could be distinguished from the wild-type population based on its flowering dynamics (Vermerris and McIntyre, 1999). The term dosage effect refers to the situation where the heterozygote has a phenotype that is distinct from both homozygotes. The data from the

Table 3 Mean height at flowering and standard deviation (SD) of the wild-type, $b m 1 /+$ and $b m / b m 1$ plants in Latin Squares IIA and IIB and the $P$-value for the comparison between height at flowering of the $b m 1 /+$ and $b m 1 / b m 1$ plants with the wild-type plants

\begin{tabular}{|c|c|c|c|c|c|c|c|c|c|c|c|c|}
\hline & \multicolumn{6}{|c|}{ Latin square IIA } & \multicolumn{6}{|c|}{ Latin square IIB } \\
\hline & \multicolumn{2}{|c|}{ wild type } & \multicolumn{2}{|c|}{$b m 1 /+$} & \multicolumn{2}{|c|}{$b m 1 / b m 1$} & \multicolumn{2}{|c|}{ wild type } & \multicolumn{2}{|c|}{$b m 1 /+$} & \multicolumn{2}{|c|}{$b m 1 / b m 1$} \\
\hline & mean & $S D$ & mean & $S D$ & mean & $S D$ & mean & $S D$ & mean & $S D$ & mean & $S D$ \\
\hline $\begin{array}{l}\text { height }(\mathrm{cm}) \\
P \text {-value }\end{array}$ & 169.4 & 19.36 & $\begin{array}{c}149.5 \\
0.0007\end{array}$ & 11.54 & $\begin{array}{l}154.0 \\
0.003\end{array}$ & 10.45 & 186.7 & 12.56 & $\begin{array}{c}187.3 \\
0.8862\end{array}$ & 10.77 & $\begin{array}{c}178.5 \\
0.0647\end{array}$ & 11.44 \\
\hline ASI (days) & -2.0 & 1.8 & -4.1 & 2.6 & -3.4 & 2.2 & -1.9 & 2.3 & -1.2 & 1.6 & -1.4 & 1.3 \\
\hline
\end{tabular}


present study with $b m 1$ heterozygotes provide evidence for a dosage effect. In the case of the Bm1 locus the heterozygote can be distinguished from the homozygotes, based on its flowering dynamics. In addition there is evidence that the composition of the cell wall is also affected in a dosage-related manner. The Bm1 locus now represents an interesting case. The $b m 1$ mutant allele is completely recessive with respect to the brown color of the midrib, partially dominant with respect to cell wall composition and overdominant with respect to flowering time. Loci that show dosage effects are not uncommon in maize. Examples include mutations affecting the endosperm (Tsaftaris et al, 1981; Singletary et al, 1997), the Pl locus, encoding a transcription factor involved in anthocyanin biosynthesis (Hollick and Chandler, 1998), and the Rp8 locus, which affects rust resistance (Delaney et al, 1998). In those cases, however, there is only one, consistent phenotypic effect.

The different analytical techniques available for the analysis of plant cell walls all have their advantages and disadvantages. The use of FT-IR spectroscopy to analyze the cell wall composition of the three genotypes has the advantage that it is relatively fast and that it does not require the isolation of individual cell wall compounds. This means that not only differences in the composition of any of the cell wall compounds can be monitored, but also differences in the chemical interactions between different cell wall compounds. Based on the FT-IR analysis, specifically because of the higher ratios of $\mathrm{A}_{1665}: \mathrm{A}_{1510}$ and $\mathrm{A}_{1595}: \mathrm{A}_{1510}$, it is apparent that the cell walls of the $b m 1$ homozygote contain higher levels of coniferaldehyde than the wild type and $b m 1$ heterozygote. This agrees with the histochemical data from the Wiesner reactions and with observations on loblolly pine (Pinus taeda L.) (Mackay et al, 1997) and tobacco (Stewart et al, 1997) in which the expression of the gene encoding CAD had been reduced. In both instances this resulted in increased incorporation of coniferaldehyde in the cell wall.

Based on the FT-IR analysis we have evidence that the cell wall of the $b m 1$ heterozygote is distinct from the cell wall of the two homozygotes. The exact chemical basis for these differences is unknown, however, because the identification of specific cell wall compounds is generally difficult with FT-IR spectroscopy. Wet chemical techniques for the determination of lignin and polysaccharide composition are more appropriate in that respect, and further studies are needed to obtain a more complete understanding of the changes in cell wall composition in the $b m 1$ heterozygote.

Variation in flowering time in brown midrib mutants provides evidence for a relationship between cell wall composition and flowering time. At this point, the chemical basis for this relationship is unknown. It is possible that one of the intermediates in cell wall biogenesis directly affects plant development. In that case the FT-IR spectra may provide clues on how the cell wall composition and flowering time are linked. Since the $b m 1$ heterozygotes flower earlier than either of the two homozygotes, correlations between wavenumbers at which the heterozygote has an absorbance distinct from the homozygotes may point towards cell wall compounds that are correlated with flowering time. A comparison of absorbances at these wavenumbers in other $b m$ mutants can reveal if this is a consistent correlation, and analysis of the cell walls with additional chemical techniques may provide further clues towards unraveling the mechanism underlying the link between cell wall composition and flowering time.

We can exclude mimicked drought stress as a basis for early flowering under the conditions tested. As far as developmental effects are concerned, at this point we can only conclude that the basis for the early flowering of the $b m 1 /+$ plants is beyond the V7 stage. Up to V7 the three genotypes develop very similarly, but it is possible that during or after the V8 stage the $b m 1 /+$ plants display accelerated growth. This needs to be investigated in more detail.

The Bm1 locus affects the expression of the enzyme CAD. Interestingly, the Cad gene was shown to affect growth in loblolly pine, and the average effect of a mutant allele, cad- $n 1$, included enhanced radial growth of the stem (Wu et al, 1999). It was suggested that the Cad locus could represent a QTL affecting stem growth in loblolly pine. The Cad locus may have a general effect on plant growth and development, although given the evolutionary distance between maize and pine, the biochemical mechanism underlying the growth effects may be different.

Finally, the fact that the $b m 1$ heterozygote has a distinct chemical and physiological phenotype may offer an additional tool for the development of new varieties. The improved forage quality of bm1 corn (Cherney et al, 1991), is not associated with increased levels of coniferaldehyde (Grabber et al, 1998). Therefore, the wild-type levels of coniferaldehyde in the $b m 1$ heterozygote do not automatically preclude the $b m 1$ heterozygote from having a potentially higher forage quality, especially since the FTIR spectrum indicates certain similarities to the $b m 1$ mutant. It may therefore be worthwhile examining the forage quality of the $b m 1$ heterozygote. In addition, other traits such as susceptibility to diseases, pests and lodging may be different in the $b m 1$ heterozygote. If the effect on flowering time is consistent in other genetic backgrounds, the data presented in this study also offer the potential to manipulate flowering time by introducing a copy of the $b m 1$ mutant allele in the line of interest.

\section{Acknowledgements}

The authors would like to thank Drs Cliff Johnston and GS Premachandra for assistance with the FT-IR spectroscopy, Dr Nick Carpita for his support, and two anonymous reviewers for helpful suggestions. This is paper no. 16698 of the Purdue Agricultural Experiment Station Series.

\section{References}

Baucher M, Monties B, Van Montagu M, Boerjan W (1998). Biosynthesis and genetic engineering of lignin. Crit Rev Plant Sci 17: 125-197.

Chabbert B, Tollier MT, Monties B, Barrière Y, Argillier O (1994). Biological variability in lignification of maize: expression of the brown midrib bm2 mutation. J Sci of Food Agric 64: 455460 .

Cherney JH, Cherney DJR, Akin DE, Axtell JD (1991). Potential of brown-midrib, low-lignin mutants for improving forage quality. Adv Agron 46: 157-198.

Delaney DE, Webb CA, Hulbert SH (1998). A novel rust resistance gene in maize showing overdominance. MPMI 3: 242245 . 
Faix O (1992). Fourier-transform Infrared Spectroscopy. In: Lin SY, Dence CW (eds) Methods in Lignin Chemistry, SpringerVerlag: Berlin. pp 83-109.

Geiger H, Fuggerer H (1979). Über den Chemismus der Wiesner-Reaktion auf Lignin. Z Naturforsch 34b: 1471-1472.

Gentinetta E, Bertolini M, Rossi I, Lorenzoni C, Motto M (1990). Effect of brown midrib-3 mutant on forage quality and yield in maize. J Genet Breed 44: 21-26.

Grabber JH, Ralph J, Hatfield RD (1998). Severe inhibition of maize wall degradation by synthetic lignin formed with coniferaldehyde. J Sci Food Agric 78: 81-87.

Halpin C, Holt K, Chojecki J, Oliver D, Chabbert B, Monties B et al (1998). Brown-midrib maize (bm1) - a mutation affecting the cinnamyl alcohol dehydrogenase gene. Plant J 14: 545-553.

Herrero MP, Johnson RR (1981). Drought stress and its effects on maize reproductive systems. Crop Sci 21: 105-110.

Hollick JB, Chandler VL (1998). Epigenetic allelic states of a maize transcriptional regulatory locus exhibit overdominant gene action. Genetics 150: 891-897.

Koester RP, Sisco PH, Stuber CW (1993). Identification of quantitative trait loci controlling days to flowering and plant height in two near-isogenic lines of maize. Crop Sci 33: 1209-1216.

Kuc J, Nelson OE, Flanagan P (1968). Degradation of abnormal lignins in the brown-midrib mutants and double mutants of maize. Phytochem 7: 1435-1436.

Lapierre C (1993). Application of new methods for the investigation of lignin structure. In: Jung G, Buxton H, Hatfield DR, RD, Ralph J (eds) Forage Cell Wall Structure and Digestibility, ASA, CSCA, SSSA: Madison, WI. pp 133-166.

Mackay JJ, O'Malley DM, Presnell T, Booker FL, Campbell MM, Whetten RW et al (1997). Inheritance, gene expression, and lignin characterization in a mutant pine deficient in cinnamyl alcohol dehydrogenase. Proc Natl Acad Sci USA 94: 8255-8260.

Moss GI, Downey LA (1971). Influence of drought stress on female gametophyte development in corn (Zea mays L.) and subsequent grain yield. Crop Sci 11: 368-372.

Neuffer MG, Coe EH, Wessler SR (1997). Mutants of Maize, Cold Spring Harbor Laboratory Press: Plainview, NY.

Provan GJ, Scobbie L, Chesson A (1997). Characterisation of lignin from CAD and OMT deficient Bm mutants of maize. J Sci Food Agric 73: 133-142.

Ribaut J-M, Hoisington DA, Deutsch JA, Jiang C, Gonzalez-de-
Leon D (1996). Identification of quantitative trait loci under drought conditions in tropical maize. 1. flowering parameters and the anthesis-silking interval. Theor Appl Genet 92: 905-914.

Sari-Gorla M, Krjewski P, Di Fonzo N, Villa M, Frova C (1999). Genetic analysis of drought tolerance in maize by molecular markers. II. Plant height and flowering. Theor Appl Genet 99: 289-295.

Séné CFB, McCann M, Wilson RH, Grinter R (1994). Fouriertransform Raman and Fourier-transform infrared spectroscopy. An investigation of five higher plant cell walls and their components. Plant Phys 106: 1623-1631.

Singletary GW, Banisadr R, Keeling PL (1997). Influence of gene dosage on carbohydrate synthesis and enzymatic activities in endosperm of starch deficient mutants of maize. Plant Phys 113: 293-304.

Stewart D, Yahiaoui N, McDougall GJ, Myton K, Marque C, Boudet AM et al (1997). Fourier-transform infrared and Raman spectroscopic evidence for the incorporation of cinnamaldehydes into the lignin of transgenic tobacco (Nicotiana tabacum L.) plants with reduced expression of cinnamyl alcohol dehydrogenase. Planta 201: 311-318.

Tsaftaris AS, Scandalios JG, McMillin DE (1981). Gene dosage effects on catalase expression in maize. J Hered 72: 11-14.

Veldboom LR, Lee M (1996). Genetic mapping of quantitative trait loci in maize in stress and nonstress environments: II. Plant height and flowering. Crop Sci 36: 1320-1327.

Vermerris W, Boon JJ (2001). Tissue-specific patterns of lignification are disrupted in the brown midrib2 mutant of maize (Zea mays L.). J Agric Food Chem 49: 721-728.

Vermerris W, McIntyre LM (1999). Time to flowering in brown midrib mutants of maize: an alternative approach to the analysis of developmental traits. Heredity 83: 171-178.

Vignols F, Rigau J, Torres MA, Capellades M, Puigdomenech P (1995). The brown-midrib3 (bm3) mutation in maize occurs in the gene encoding caffeic acid O-methyl transferase. Plant Cell 7: 407-416.

Whetten RW, MacKay JJ, Sederoff RR (1998). Recent advances in understanding lignin biosynthesis. Annu Rev Plant Physiol Plant Mol Biol 49: 585-609.

Wu RL, Remington DL, MacKay JJ, McKeand SE, O'Malley DM (1999). Average effect of a mutation in lignin biosynthesis in loblolly pine. Theor Appl Genet 99: 705-710. 